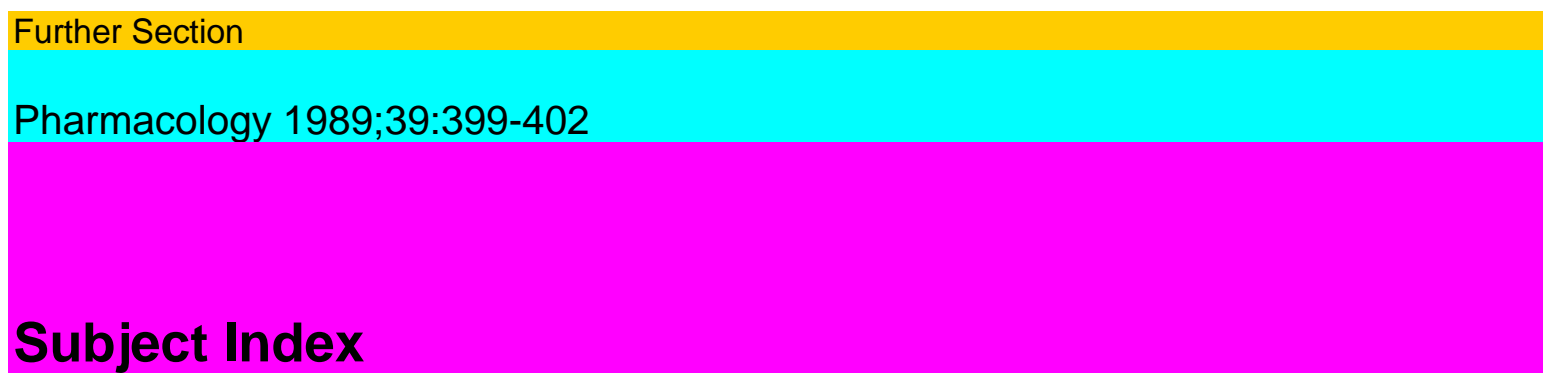

Acetone 137 Acetylation 39

Acetylcholinesterase 154, 367 Acteyltransferase 299 Acid production 265 Acidosis, myocardial 1 Aconitine 103

Adenylate cyclase $121,165,390$ Adipose tissue 89 Adrenal tissue 89 Adrenalectomy $28 \alpha$ Adrenergic agonists $240 \alpha$-Adrenergic antagonists $192 \mathrm{c}^{1 / 8}$-Adrenergic receptors 240

Adrenoceptors 327, 379 ai-Agonists 176 Allergic mediators 19 Allograft recipients 160

Alloxan 291 Alprazolam 129 Amaryllidaceae alkaloid 50 Amiloride 230 Aminopyrine 299 accumulation 265 5-Aminosalicylic acid 39 Analgesic tolerance 145 Anaphylaxis 19

Anesthesia 383

Angiotensin II 11, 115, 121, 330 Anococcygeus muscle, rat 115 Anthraquinone drugs 362 Antibiotics 213 Antidepressant drugs 285 Antihypertensives 109 Anti-inflammatory activity 103 Anti-inflammatory drugs 350 Antimuscarinic activity 154 Antioxidants 39 Antrum 98 Aorta 11,317,327,377 Aortic rings 176, 177 Arachidonic acid 291

Arginine-vasopressin 11 Arrhythmia 11 Arterial pressure 1 Arthritis 98 Arthus reaction 28

Ascaris suum extract 20 Assay, catecholamines 242 -, glutathione 206 -, tin 225 Asthma 19

Atenolol $109(\mathrm{H}+-\mathrm{K}+)$-ATPase 11 Atropine 70, 154,253 Azathioprine 30

Barbiturates 383 Basolateral membranes 121 Basophils 19 Behavioral responses 46

Benzo(a)pyrene hydroxylase 89 Benzodiazepines 129 Benzothiazolamine 11 Benzyl alcohol 165 Bethanechol 69 Bile pigments 224 Biliary secretion 160 Bilirubin 224, 273 Bladder 69 ßBlockers 109 Blood glucose 294

- $\quad$ pressure 109,292

Blood-brain barrier 367

Body weight 294

Bradycardia 109

Bradykinin 19

Brain 285

Brattleboro rat 165,240

Breast 89

Calcium 11,69,327

channel 11, 327

- blockers 309

ionophoreA23187 19

uptake 317 Calmidazolium 317 Calmodulin 309,317

antagonists 317 cAMP 121, 165,265 Canabinoids 337 Carbachol 165,327 Carcinogens 89, 137 Cardiac death 129

glycosides 11

myocytes 230 Carrageenin-induced paw edema 
30, 103 Carvedilol 327 -, chemical structure 328 Cartilage 362 Casein 90 Cataract 59

Cataractogenesis 59 Catecholamine stores 240 Catecholaminergic systems 109 Cefazolin 213 Cefotetan 213 Cefpiramide 213 Celiprolol 1 Cell aggregates 231

culture 231

isolation 351 Cell-mediated immunity 28 Cells, cultured brain 367 Cerebellum 285 Cerebral cortex 145 Children, latency-age 46 Choline acetyltransferase 367 Choline-bitartrate 90

Cholinergic enzymes 367

interaction 200 Ciclosporin 160, 205 Cigarette smoke 89 Cimetidine 200 Clonidine 109,240 400

Subject Index

CNS 154 Colonocytes 39 Contractile function 192

- responses, arginine-vasopressin

13

- serotonin 13 Contraction 11, 185, 317 Corn oil 89 Coronary artery 1

flow 1 Corpus 98 Cortex 285, 299 Creatinine clearance 160 Culture 230 Cultured brain cells 367 Cyclooxygenase 291 Cyclophosphamide 30 Cytochrome P-450 89, 137, 273

creductase 89

Dazmegrel 383 DbcAMP 265 O-Deethylase 299 N-Deethylase 299 Depolarization 11,185 Desensitization 115 Detoxication 90,299 Dexamethasone 362 Diabetes mellitus 59 Diabetic rats 291 Diacerein 362 Dichlorobenzamil 230 Diet 89 Dietary fat 89 Digitalis glycosides 230 Dihydropyridine receptors 309 Diisopropylfluorophosphate 154 Diltiazem 70, 309

Dimethylbenz(a)anthracene 89 Dimethylsulphoxide 98 Disodium cromoglycate 30 Diuresis 240 Diuretics 230 DMSO 98 Dopamine 178,318 Drug-metabolizing enzymes 89

Electrical field stimulation 185 Endocardiogram 1 Endothelium 373

Energy intake 91 Epigalanthamine 50 Epithelial cells 39 Epoxide hydrolase 299 Ethanol 367 Ethoxycoumarin 299 7-Ethoxycoumarin deethylase 273 Ethoxyresorufin O-deethylase 299

Famotidine 200

Fat 89

Fatty acid oxidation 39

Fetal alcohol syndrome 367

blood 367

brain 367 Fetus 367 5-Fluorouracil 78 Formazans 103 Forskolin 19 Forssman shock 30

Galanthamine hydrobromide 50 Gastrointestinal ulceration 98 Glomerular filtration 160

Glucuronyl transferase 299 Glutathione 205,213,273

content, brain 281

depletion 205

peroxidase 299 Glyoxalase 299 Guanabenz 240 Guanfacine 240

Heart, chick embryo 230

rate 1, 109 Hemagglutination 337

titer assay 340 Heme 224

oxygenase 224, 275 Hemodynamics 1 Hepatotoxicity 205 Hippocampus 285 Histamine 265

$7 / 8$-receptor antagonists 200

receptors 318 5-HT receptors 145 Human uterus 309 Hydroxytryptamine 145,373

Hypersensitivity 28 Hypertension 240, 383

Hypotension 291 Hypothalamus 285 
Immune response 337

suppression 337

system 350

Immunosuppressive agent 28, 205 Indicator-dilution technique 253 Indocyanine green 213 Indomethacin 98, 115, 291, 350

- $\quad$ ligand association assay $35 \Gamma$

Inflammation 28, 103, 350

Inorganic metals 224

Insulin 291

Intestinal epithelium 224

- $\quad$ heme oxygenase 224

Intestine 89

Ischemia 1, 129

I schemic arrest 129 Isolated colonocytes 40

rat heart 129

- parietal cells 265

seminal vesicle 185 Isoproterenol 19,327

Kappa opioid receptor 145 Ketanserin 145 6-Keto-PGFia 291 Kidney 89, 121, 160, 165,205, 224,299

B-Lactam antimicrobials 213 Latency-aged children 46 LDH, determination 352 Lens fiber cells 59 Leucocyte 350 Leukotriene C4 19 Lidocaine 265 Ligandin 213 Liver 89,

$137,205,224$

dysfunction 160

glutathione S-transferase 213 Local anesthetics 265 Long-Evans rats 240

LTC4 19 Lung 253

Macrophages 362 Marihuana 337 Mast cell 19

Subject Index

401

Medulla, kidney 299 Mental retardation 367 Metabolism 69,273

- ciclosporin 160

Metalloporphyrin 224, 273

Metaraminol 178

L-Methionine 90

Methotrexate 79

$\alpha$-Methyldopa 109

Methylphenidate 46

Mianserin 285

Microsomal cytochrome P-450 137

heme 224

vesicles 265 Microsomes 137

Mixed function oxidase 137, 300 Monensin 357 Monoclonal antibodies 137 Mouse end-plate 200 Moxalactam 214 Mucosal lesions 98 Muscarine 318 Muscarinic receptors 69,253

stimulation 69 Muscle, anococcygeus 115 Mutagens 89 Myocardial acidosis 1

cells 230

ischemia 129 Myocytes 230 Myo-inositol 59 Myometrial tissue 310 
$\mathrm{Na}+$ transport 231

NADPH cytochrome c reductase

299 Naloxone 109 Natriuresis 240 Neonatal mouse 390 Nephrectomy 299 Nephrotoxicity 205 Neuromuscular junction 200 Neurotransmitters 185 Nicotinamide 69 Nifedipine 309

Nitrite 39 Nitrosamine 137 Nivalin 50 Noradrenaline 115,178,185,240, 373

Opioides 109 Organ transplantation 160 Organometallic compounds 224 Osteoarthritis 362 Osteoblast-like cells 390 Ouabain 230, 373

- $\quad$ intoxication 11

Oxadiazole-thiones 103

Oxygenase 224

PAF 19

Pain 98, 103

Papaverine 185

Papillary muscle 11

Parasympathetic stimulation 69

Parathyroid hormone 121,390

Parietal cells 265

Passive Arthus reaction 28

- $\quad$ cutaneous anaphylaxis 19

Patients 300

Paw oedema 105 Pentobarbital 383 Peptide mapping 141 Phagocytosis 362

Pharmacokinetics, 5-fluorouracil 78

- galanthamine hydrobromide .

Phase-I enzymes 299

Phase-II enzymes 299

Phenolamine 115, 185,329

Phenylephrine 178,373

Picryl chloride 30 Pirenzepine 253 Pituitary 89

Plasma vasopresin levels 166 Platelet-activating factor 19 Poisoning, anticholinesterase activity 154 Polymo $\varphi$ honuclear leucocytes 3 Porphyrins 273 Prazosin 192 Prednisolone 30 Pregnant human uterus 309 Pressure 383 Probenecid 357 Propranolol 1, 19,329 Propyleneglycol 383 Prostacyclin 291 Protopo $\varphi$ hyrin 224 Psoriasis 78

Pulmonary artery 383

endothelium 253

hypertension 383

microvasculature 253

vascular resistance 383 Purinic neurones 185

Ranitidine 200 Rauwolscine $374 \alpha$-Receptor 176 Receptor regulation 165

subtypes 253 Receptors, Mi 253 Relaxation 327 Renal cortex 299

function 240

- tubular basolateral membranes

121

Reperfusion 129

Resepine 177

Reversed passive Arthus reaction 
30 Rubidium 185

Salazosulfapyridine 39

Salbutanol 19

Sahcylate derivatives 39

Saphenous vein 327

Seminal vesicle 185

Sensitization 28

Septum 285

Serotonin 11, 19, 318, 374

Sheep 383

Silicone oil cushion centrifugation

350 Skin 98

reactions 19 Small intestine 98,224 Smooth muscle 19,70 Sodium entry 230

excretion 240

nitroprusside 291 Spinal cord 145 Spleen 337

cell counts 340

weight assay 340 Stenosis 1 Stomach 98, 265 Streptozocin 59 Stress 98

402

Subject Index

Sulphotransferase 299 Swelling 98

Tachyphylaxis 115 Temperature 115 Theophylline 19 Thioglycollate 362 Thioltransferase 299 Thiomethyltransferase 299 Thiopuriemethyltransferase 299 Thoracotomy 2

Thromboxane 383

\section{A2 291}

B2 291 Tin 273

tissue levels 227 Tin-protoporphyrin 224, 273

Tolazoline 178 Tolerance 147 Trachea 327 Transport 213 Trifluoperazine 309,317 Tumor, kidney 299

Ulcer 98, 104 - index 98 Ulcerative colitis 39 Urethra 192 Uridine-5'-diphosphoglucuronic acid 89 Urinary excretion 160 Urine 160,240 Uterine action 390 Uterus 309, 327

Valinomycin 357 Vascular permeability 19

resistance 383 Vasoconstriction 327, 373 Vasoconstrictor agents 373 Vasodilators 327

Vasopressin 240

receptor 165, 240 Ventricular arrhythmias 129 Verapamil 309

Volume retention 240

Writhing 103 Yohimbine 109, 192 